months after onset of seizures, whereas those not treated with VPA lived for 7 to 16 months. (Bicknese AR et al. Early childhood hepatocerebral degeneration misdiagnosed as valproate hepatotoxicity. Ann Neurol Dec 1992; $\underline{32:}$ 767-775). (Correspondence: Dr WE Dodson, St Louis Children's Hospital, 400 S Kingshighway Blvd, St Louis, MO 63110).

COMMENT. The authors propose that many of the reported patients with VPA-associated hepatotoxicity represent undiagnosed hepatocerebral degeneration, the Huttenlocher variant of Alpers' syndrome. Their experience suggests that liver failure is accelerated by exposure to VPA, and an alternative antiepileptic treatment should be employed in young children with resistant seizures and mental and motor regression, indicative of cerebral degeneration. Recurrence of this syndrome in family members suggests an autosomal recessive inheritance, but the biochemical basis remains undetermined. Of 13 similar cases reported from the Hospital for Sick Children, London (Egger J et al. Clin Pediatrics 1987; 26: 167), only 4 had received VPA and 2 may have died from VPA-hepatotoxicity.(See Ped Neur Briefs July1987)

\title{
NEURODEGENERATION WITH TRICHORRHEXIS INVAGINATA
}

Two siblings are reported from the Medical College of Ohio with an autosomal recessive syndrome characterized by hair and skin abnormalities, hypoplastic nails, hypotonia, areflexia, and progressive neurological deterioration. Mutiple abnormalities were observed at birth, and apneic episodes secondary to laryngomalacia occurred in the neonatal period. At 10 months, CT showed early cortical atrophy, and EEG revealed seizure activity and encephalopathy. At 12 months, neuromotor deterioration had progressed and the child was no longer able to suck, did not smile, and did not move her legs. Apneic episodes and respiratory difficulties worsened and she died at 16 months. The sibling with a similar syndrome was alive at 27 months. (Gyure KA et al. Autosomal recessive neurodegenerative disorder with trichorrhexis invaginata and ectoderma dysplasia. Pediatr Neurol Nov/Dec 1992; $\underline{8}$ : 469-72). (Correspondence: Dr TW Kurczynski, Dept of Pediatrics, Medical College of Ohio, PO Box 10008, Toledo, $\mathrm{OH} 43699)$.

COMMENT. Trichorrhexis invaginata, or bamboo hair, is an hair-shaft defect usually found with ichthyosiform dermatoses and Nethertons syndrome. Its significance in relation to the present neurodegenerative disorder is unknown. Hair-shaft abnormalities occur as a feature of various neurological diseases, including Menkes disease, biotin deficiency, and Pollitt syndrome. Electron micrographs of hair samples may be of diagnostic importance in children with neurologic disorders. 